\title{
Incidence and prognosis of intra-abdominal hypertension in critically ill medical patients: a prospective epidemiological study
}

Patricia Santa-Teresa ${ }^{1 *}$, Javier Muñoz ${ }^{1}$, Ignacio Montero ${ }^{1}$, María Zurita', María Tomey ${ }^{1}$ Luis Álvarez-Sala², Pablo García ${ }^{1}$

\begin{abstract}
Introduction: The aim of this study was to determine the incidence of intra-abdominal hypertension (IAH) in patients with two or more categorized risk factors (CRF) for IAH, and their morbidity and mortality during their intensive care unit (ICU) stay.

Methods: Prospective cohort study carried out at a medical ICU. A total of 151 medical patients were enrolled during a period of 3 months. After ICU whole staff training, we conducted daily screening of the four CRF for IAH based on the World Society of Abdominal Compartment Syndrome (WSACS) guidelines (namely, diminished abdominal wall compliance, increased intraluminal content, increased abdominal content, and capillary leak syndrome or fluid resuscitation). In those patients with risk factors of at least two different categories ( $\geq 2$ CRF), intra-abdominal pressure (IAP) was measured every $8 \mathrm{~h}$ during ICU stay. Data included demographics, main diagnosis on admission, severity scores, cumulative fluid balance, daily mean IAP, resolution of IAH, days of ICU and hospital stay, and mortality.

Results: Eighty-seven patients (57.6\%) had $\geq 2$ CRF for IAH, 59 (67.8\%) out of whom developed IAH. Patients with $\geq 2$ CRF had a significantly higher mortality rate (41.4 vs. $14.3 \%, p<0.001)$. Patients with IAH had higher body mass index, severity scores, organ dysfunctions/failures, number of CRF for IAH, days of ICU/hospital stay and hospital mortality rate ( 45.8 vs. $32.1 \%, p=0.22$ ). Non-resolution of IAH was associated with a higher mortality rate (64.7 vs. $35.3 \%, p=0.001)$. None of the cohort patients developed abdominal compartment syndrome. The multivariate analysis showed that IAH development (odds ratio (OR) 4.09; 95\% confidence interval (CI) 0.83-20.12) was a nonindependent risk factor for mortality, and its non-resolution (OR 13.15; 95\% Cl 22.13-81.92) was an independent risk factor for mortality.
\end{abstract}

Conclusions: Critically ill medical patients admitted to ICU with $\geq 2$ CRF have high morbidity, mortality rate, and incidence of IAH, so IAP should be measured and monitored as recommended by the WSACS. Our study highlights the importance of implementing screening and assessment protocols for an early diagnosis of IAH.

\section{Introduction}

Intra-abdominal hypertension (IAH) is associated with adverse outcomes in critically ill patients [1,2]. Elevated intra-abdominal pressure (IAP) above physiologic limits has deleterious effects on end-organ function due to pathophysiologic changes such as a drop in cardiac

\footnotetext{
* Correspondence: patriciasantateresa@hotmail.com ${ }^{1}$ Intensive Care Unit, Hospital General Universitario Gregorio Marañón, Calle Doctor Esquerdo 46, Madrid, 28007, Spain

Full list of author information is available at the end of the article
}

output, diminished chest wall compliance, decreased renal and visceral blood flow, and increased intracranial pressure [3-11].

IAH has traditionally been associated with patients undergoing abdominal surgery and trauma patients who require aggressive volume resuscitation [12-16]. Several studies have demonstrated that IAH is also frequently present in critically ill nonsurgical patients, and is now considered to be associated with the general process of inflammation and resuscitation [17-22]. However,

\section{SpringerOpen ${ }^{\circ}$}

(C) 2012 Santa-Teresa et al.; licensee Springer This is an open access article distributed under the terms of the Creative Commons Attribution License (http://creativecommons.org/licenses/by/2.0), which permits unrestricted use, distribution, and reproduction in any medium, provided the original work is properly cited. 
establishing comparisons for incidence, morbidity and mortality rates in medical patients from data of published clinical trials is difficult, mainly due to three reasons: firstly, the absence of uniform definitions before consensus guidelines, which allow us to reproduce and interpret the results; secondly, the different methods used for IAP measurement; and lastly, the fact that most clinical studies have been performed on mixed populations (both medical and surgical).

The World Society of Abdominal Compartment Syndrome (WSACS) has published a consensus statement including definitions and recommendations for the screening and management of IAH and abdominal compartment syndrome (ACS) [23,24]. Following this consensus, the aim of this study was to determine the incidence of IAH in exclusively critically ill medical patients with high risk of IAH development, and their morbidity and mortality during their intensive care unit (ICU) stay.

\section{Materials and methods}

This study was conducted in a university hospital medical ICU in accordance with a protocol that was approved by the local ethics committee. The study fulfilled the requirements of the Declaration of Helsinki, and included an informed consent from the patients on admission (or their family if necessary) to participate and publish the results.

All medical patients admitted to the ICU during a three-month period and expected to stay $>24 \mathrm{~h}$ were prospectively enrolled, provided they needed a bladder catheter. Exclusion criteria were ICU stay $<24$ h, age $<$ 18 years, pregnancy, contraindication for intravesical pressure measurement (pelvic fracture, hematuria, or neurogenic bladder), and bladder surgery. WSACS consensus for assessment of IAH was implemented as a new protocol $[23,24]$.

\section{Data collection}

\section{Demographic data}

Age, gender, body mass index (BMI) expressed as $\mathrm{kg} / \mathrm{m}^{2}$ [25], main diagnosis on admission, cumulative fluid balance, length of ICU and hospital stay, and hospital mortality were considered in collecting the patients' data. Patients were followed until death or hospital discharge, whatever came first.

\section{Organ dysfunction}

On admission, APACHE II (Acute Physiology and Chronic Health Evaluation) and SOFA (Sequential Organ Failure Assessment) scores were recorded using the worst values of the day [26,27]. Organ dysfunction/ failure was evaluated using the SOFA score (dysfunction $=$ subscore $\leq 2$, failure $=$ subscore $\geq 3$ ). The SOFA maximum score was based on the worst value of each organ system during the ICU stay.

\section{Risk factors for IAH}

All patients were prospectively screened for risk factors (RF) of IAH on admission and daily during their ICU stay until death or discharge, based on four categories from the WSACS algorithm (Table 1). Those patients who presented any RF of at least two different categories $(\geq 2$ categorized risk factors (CRF)), at any time on admission or during the ICU stay, were considered to have a high risk of developing IAH, and IAP was monitored (starting the day they were found to have $\geq 2$ CRF). Those patients with $<2$ CRF on admission and daily during the ICU stay were considered to have a low risk of developing IAH according to WSACS recommendations and their IAP measurement was not recorded. For the main analysis, we calculated the number of RF as categories (one minimum, four maximum) on admission (first $24 \mathrm{~h}$ ) and during the ICU stay (mean RF categories present per day).

\section{IAP measurement}

IAP was measured intravesically using a closed-system Foley bladder catheter according to the modified Kron technique $[28,29]$ with an instillation volume of $25 \mathrm{ml}$ of saline. Abdominal perfusion pressure (APP) was calculated as mean arterial pressure minus IAP $[30,31]$. Measurements of IAP and APP were recorded every $8 \mathrm{~h}$ in patients with $\geq 2$ CRF until resolution of IAH (in case it developed), death, or discharge from the ICU. IAH was defined as a repeated (at least three consecutive values separated by $8 \mathrm{~h}$ ) elevation of $\mathrm{IAP}_{\min }$ (the lowest value of each of the three IAP measures) $\geq 12 \mathrm{mmHg}$ [23]. For the main analysis, $\mathrm{IAP}_{\text {mean }}$ and $\mathrm{APP}_{\text {mean }}$ (mean of the three daily values) were calculated per day.

\section{IAH characteristics}

We recorded the day of onset, days of sustained IAH, surgical abdominal decompression, and resolution. Resolution of IAH was defined as a sustained IAP $\mathrm{Imin}_{\text {min }}<12$ $\mathrm{mmHg}$ in all measurements recorded every $8 \mathrm{~h}$ for a minimum of $48 \mathrm{~h}$; and non-resolution was defined as a sustained $\mathrm{IAP}_{\min } \geq 12 \mathrm{mmHg}$ in all measurements until death or discharge from the ICU. IAH was classified according to the time of onset (on admission or during ICU stay) and the development of symptoms (acute, over a period of hours; subacute, over a period of days; or chronic, over a period of months/years). We also recorded the grade of maximal IAP reached during the ICU stay (I, 12-15; II, 16-20; III, 21-25; IV, $\geq 25 \mathrm{mmHg}$ ) [23].

\section{Statistical analysis \\ Univariate analysis}

Continuous non-normally distributed variables were expressed as the median and interquartile range and compared using the nonparametric Mann-Whitney test. Continuous normally distributed variables were 


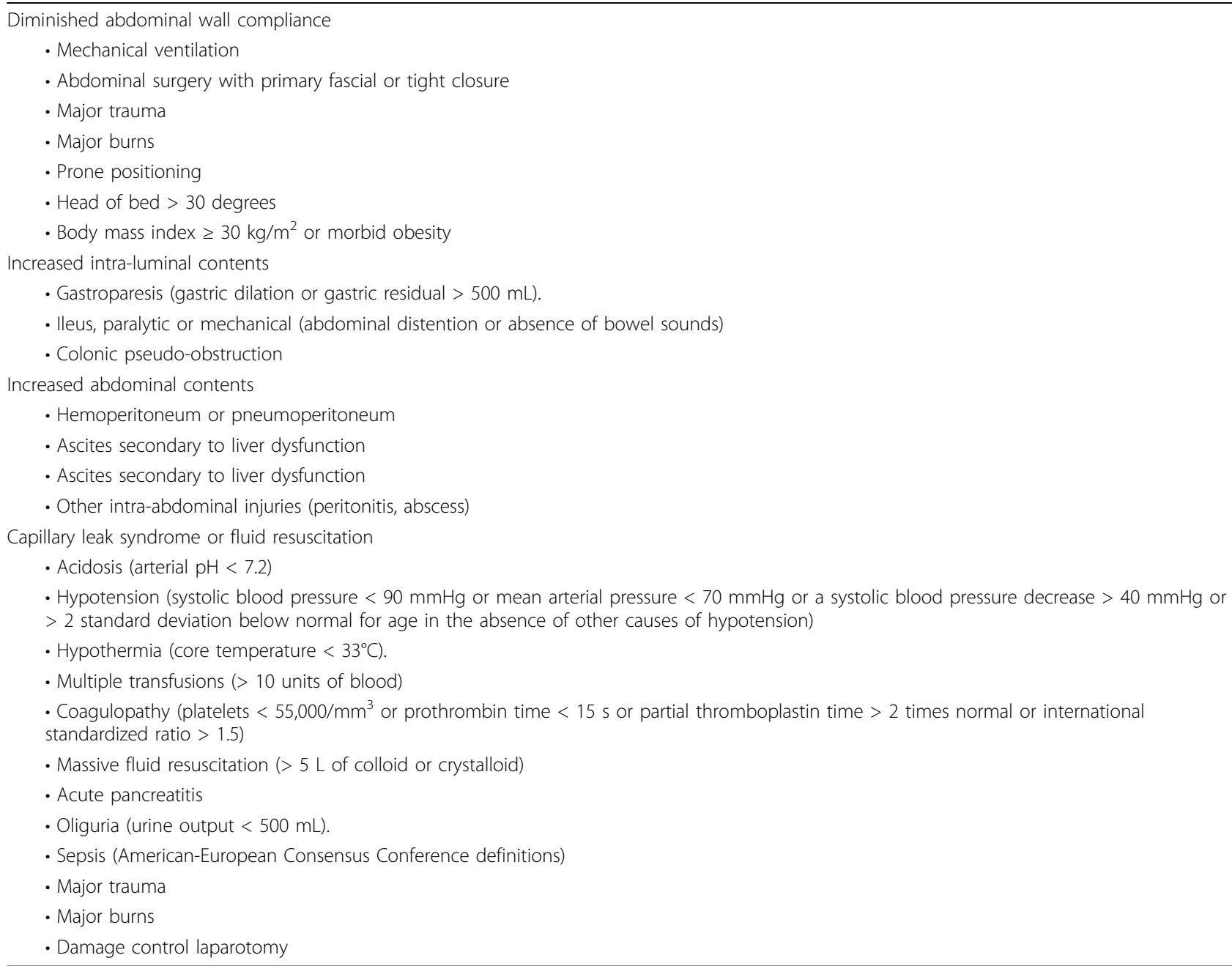

expressed as mean \pm standard deviation, and compared using the student's $t$ test. Categorical variables were expressed as numbers and percentages and compared using the chi-square test or Fisher exact test. All $p$ values were two-tailed, and $p<.05$ was considered as statistically significant.

\section{Multivariate analysis}

The prognostic relevance of two different parameters was analyzed: variables associated with development of IAH and the prognostic value of IAH and its non-resolution. Backward multiple logistic models were used, including all the variables yielding $p<.2$ by univariate analysis and those considered clinically relevant. Possible interactions were tested. The results were summarized as odds ratios their respective $95 \%$ confidence intervals. The area under the receiver operating characteristic (ROC) curve was used to assess discrimination; an area under the ROC curve of 1.0 denotes perfect discrimination, whereas a value close to 0.50 indicates no apparent accuracy. The Hosmer-Lemeshow goodness-of-fit test was used to evaluate agreement between the observed and expected results across all the probability strata of the outcome of interest (calibration); $p>.05$ indicates a good fit for the model. Kaplan-Meier curves for survival at 28-day hospital stay were constructed for subgroups with IAH.

\section{Results}

Criteria for IAP monitoring and incidence of IAH (Figures 1 and 2)

During the study period, 204 patients were admitted. Fifty-three were excluded: 5 refused to participate, 22 had ICU stay < 24 hours, 12 had contraindications for intravesical pressure measurement or bladder surgery, 11 did not require urinary catheterization, 2 were $<18$ years and 1 was pregnant. We finally enrolled 151 medical patients, and the entire protocol was completed in all of them. Main diagnosis on admission was 


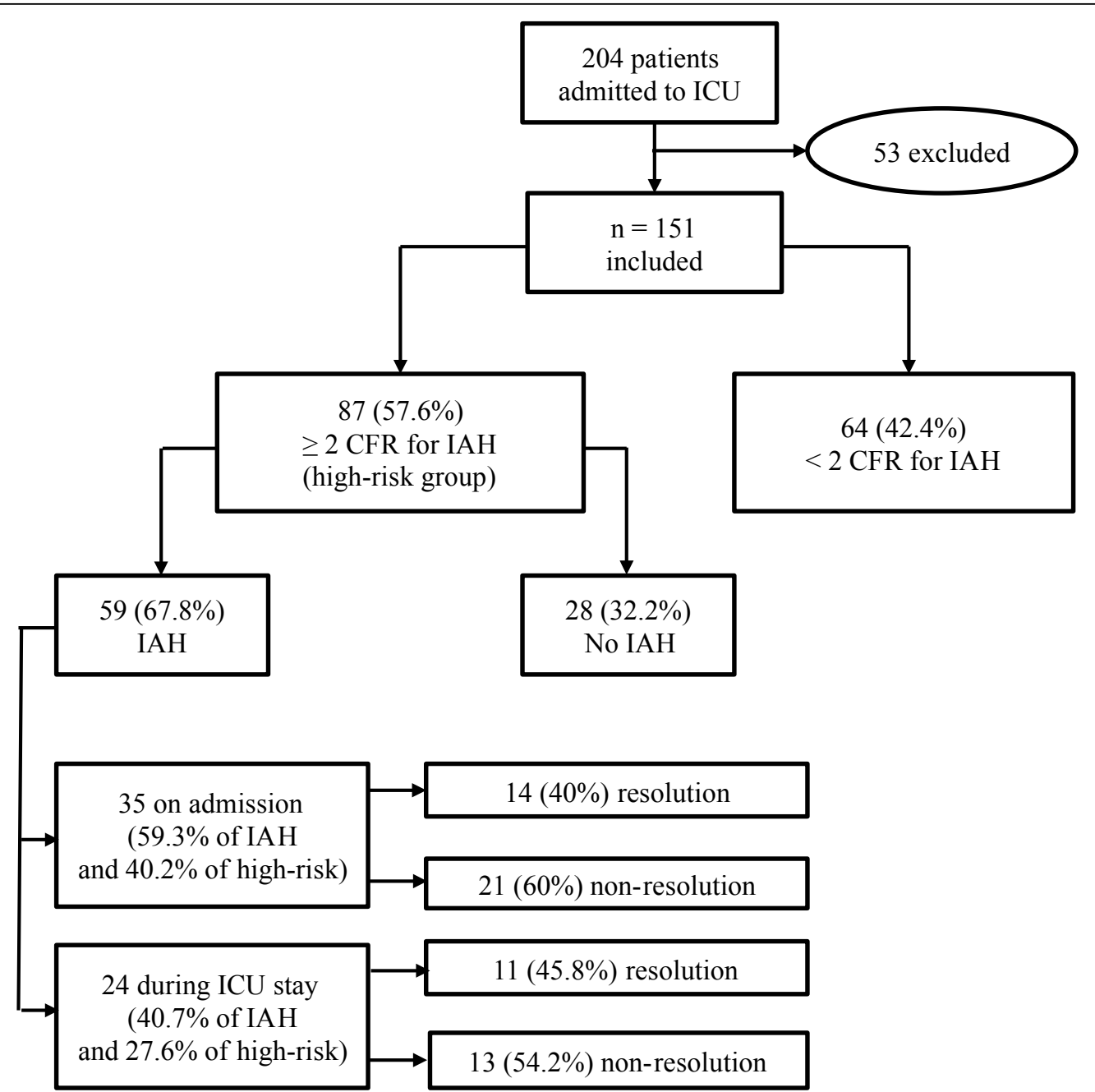

Figure 1 Algorithm and general results of the study cohort. IAH, intra-abdominal hypertension; ICU, intensive care unit; CRF, categorized risk factors.

cardiopulmonary disease (Table 2). Sixty-four patients presented $<2$ CRF (42.4\%) on admission and during all the ICU stay, and IAP was not measured; the remaining 87 (57.6\%) patients presented $\geq 2$ CRF (high risk group) for IAH (88.5\% on admission and $11.5 \%$ during ICU stay), and IAP was monitored following the study protocol. In the high risk group, 59 of the 87 patients developed IAH (67.8\%): 35 on admission (59.3\%) and 24 during the ICU stay (40.7\%). Overall $67.8 \%$ of the high risk group subjects developed IAH, $40.2 \%$ on admission, and another $27.6 \%$ during the ICU stay. Figure 2 shows the differences in the evolution of the number of CRF during the ICU stay across different subgroups of patients. Comparisons between survivors and non-survivors in the study cohort and high risk group are shown in Tables 3 and 4. There were no statistical differences in IAP levels between survivors and non-survivors (11.8 vs. $12.0 \mathrm{mmHg}, p=0.78$ ).
General characteristics for the comparison between high risk and low risk groups (Table 5)

We compared patients with $\geq 2$ CRF $(n=87)$ with those with $<2$ CRF for IAH $(n=64)$. Severity of illness, ICU and hospital length of stay, and mortality rate (41.4 vs. $14.3 \%, p<0.001)$ were significantly higher in the high risk group.

General characteristics for the comparison between IAH and non-IAH groups (Tables 6 and 7)

We compared patients who developed IAH with $\geq 2$ CRF $(n=59)$ with those who did not despite having $\geq 2$ CRF $(n=28)$. Mortality rate was higher in patients with IAH, although this did not reach statistical ( 45.8 vs. $32.1 \%$, $p<0.22)$ significance. Patients with IAH were older, had a higher BMI, higher severity scores and proportion of organ failure, and had a longer ICU and hospital stay. Increased abdominal content (39 vs. $10.7 \%, p=0.007$ ) 
$\mathbf{A}$

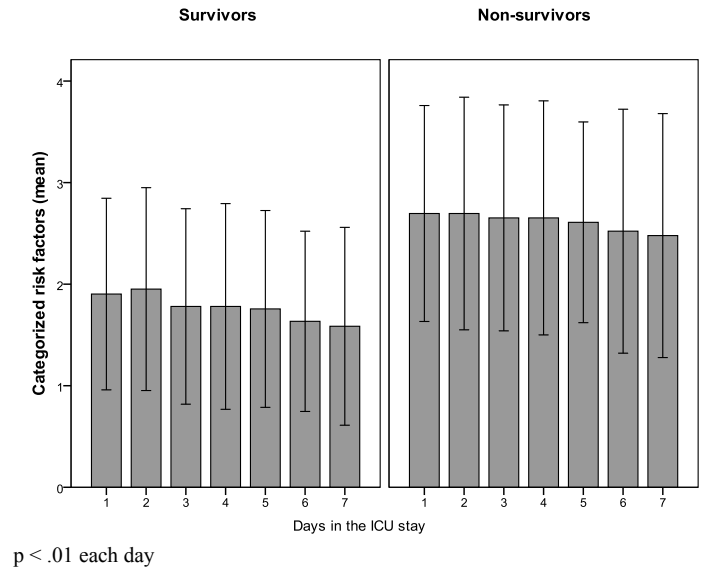

B

IAH Non-AH

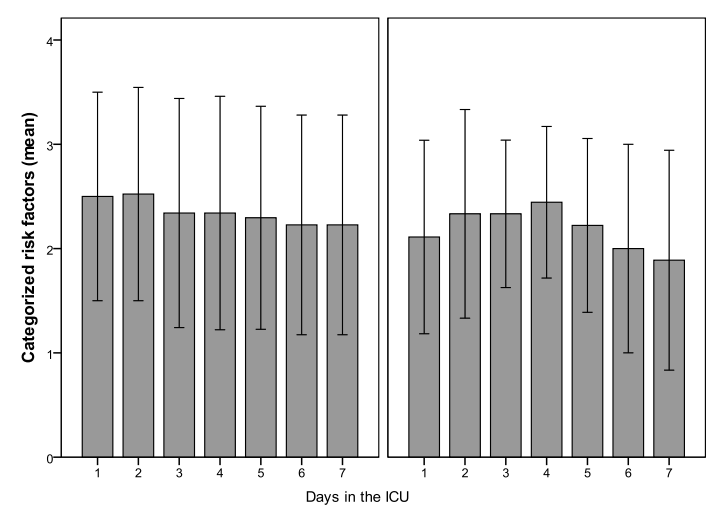

$\mathrm{p}>.05$ each day

C

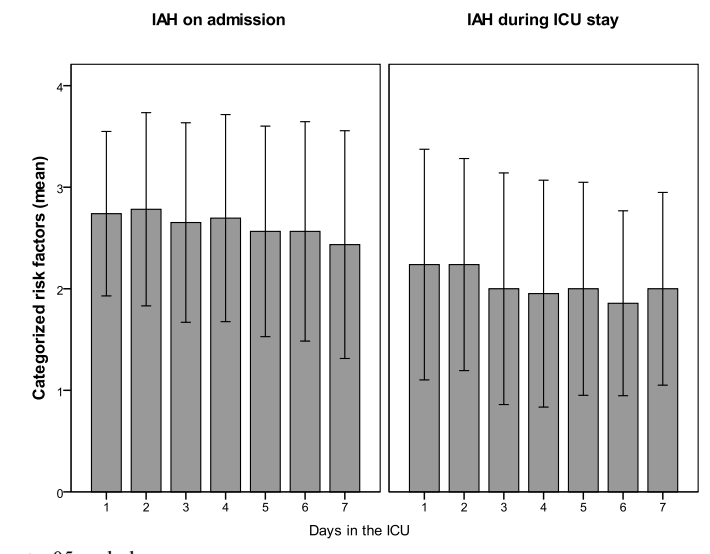

$\mathrm{p}>.05$ each day

Figure 2 Differences in the evolution of the number of categorized risk factors during the ICU stay. Differences in the evolution of the number of categorized risk factors during the ICU stay across survivors and non-survivors in the study cohort (A), patients with and without IAH (B), and patients with IAH on admission or during the ICU stay $(\mathbf{C})$. ICU, intensive care unit; IAH, intra-abdominal hypertension.
Table 2 Main diagnosis of the study cohort on admission

\begin{tabular}{lc}
\hline & Study cohort $(\boldsymbol{n}=\mathbf{1 5 1})$ \\
\hline Cardiopulmonary diagnosis & $67(44.4)$ \\
Acute/chronic liver failure & $7(4.6)$ \\
Acute pancreatitis & $5(3.3)$ \\
Gastrointestinal disease & $8(5.3)$ \\
Other intra-abdominal processes & $4(2.6)$ \\
Acute renal failure & $13(8.6)$ \\
Cardiac arrest & $5(3.3)$ \\
Neurologic disease & $30(19.9)$ \\
Other & $12(8)$ \\
\hline
\end{tabular}

Values are $n$ (percent).

and $\operatorname{IAP}_{\text {mean }}(13+1$ vs. $8+1 \mathrm{mmHg}, p<0.001)$ were also significantly higher.

\section{General characteristics of patients with IAH (Table 8, \\ Figure 3)}

Non-survivors had significantly higher severity scores, number of organ failures, and CRF for IAH, both on admission and during the ICU stay. The more commonly found subtypes of IAH were subacute (51 patients, $86.4 \%$ ) and grade II (34 patients, 57.6\%). IAH was resolved in a high proportion of patients (42.4\%) and none presented ACS or required surgical abdominal decompression. Patients in whom IAH was not resolved (57.6\%) had a significantly higher mortality rate than patients whose condition was resolved (64.7 vs. $35.2 \%, p$ $=0.001$ ). Kaplan-Meier survival analysis for patients with IAH on admission or during the ICU stay depending on resolution is shown in Figure 3.

\section{Multivariate analysis: predictive and prognostic models (Tables 9, 10 and 11) \\ Predictive model for IAH development in the high risk group (87 patients with $\geq 2$ CRF)}

SOFA on admission, obesity, and number of CRF during ICU stay (odds ratio (OR), 2.31; 95\% confidence interval (CI), 1.09 - 4.88) were independent predictors of IAH development.

Predictive model of mortality in the high risk group (87 patients with $\geq 2$ CRF)

IAH development was a non-independent predictor of mortality (OR, 4.09; 95\% CI, 0.83 - 20.12).

Predictive model of mortality in the IAH group (59 patients) Non-resolution of IAH (OR, 13.15; 95\% CI, 22.13 81.92), number of CRF during ICU stay (OR, 9.94; $95 \%$ $\mathrm{CI}, 1.59-62.00)$, and age $\geq 65$ years were independent predictors of mortality. Even though the ROC curve showed good discrimination, the Hosmer-Lemeshow test indicated inadequate calibration across all the probability strata for mortality in the model. 
Table 3 Categorized risk factors between survivors and non-survivors in the study cohort

\begin{tabular}{|c|c|c|c|}
\hline & Survivors $(n=109)$ & Non-survivors $(n=42)$ & $p$ value \\
\hline \multicolumn{4}{|l|}{ Number of categorized risk factors } \\
\hline On admission & $1.5 \pm 0.9$ & $2.4 \pm 1.1$ & $<.001$ \\
\hline During ICU stay & $1.6 \pm 1$ & $2.8 \pm 1.1$ & $<.001$ \\
\hline \multicolumn{4}{|l|}{ Risk factors during ICU stay } \\
\hline \multicolumn{4}{|l|}{ (n, percent) } \\
\hline Diminished abdominal wall compliance & $75(68.8)$ & $39(92.8)$ & $=.002$ \\
\hline Increased intraluminal contents & $20(18.3)$ & $24(57.1)$ & $<.001$ \\
\hline Increased abdominal contents & $10(9.2)$ & $16(38.1)$ & $<.001$ \\
\hline Capillary leak syndrome/fluid resuscitation & $61(55.9)$ & $36(85.7)$ & $=.001$ \\
\hline
\end{tabular}

ICU, intensive care unit. Data are presented as mean \pm standard deviation, unless otherwise indicated.

Table 4 Categorized risk factors between survivors and non-survivors in the high risk group

\begin{tabular}{lccc}
\hline & Survivors $(\boldsymbol{n}=\mathbf{5 1})$ & Non-survivors $(\boldsymbol{n}=\mathbf{3 6})$ & \\
\hline Number of categorized risk factors & & & \\
$\quad$ On admission & $2.2 \pm 0.8$ & $2.7 \pm 0.9$ & $<.001$ \\
$\quad$ During ICU stay & $2.6 \pm 0.7$ & $3.1 \pm 0.8$ & \\
Risk factors during ICU stay (n, percent) & & & \\
$\quad$ Diminished abdominal wall compliance & $48(94.1)$ & $35(97.2)$ & $<.001$ \\
Increased intraluminal contents & $20(39.2)$ & $24(67.6)$ & $<.001$ \\
Increased abdominal contents & $10(19.6)$ & $16(44.4)$ & $<.001$ \\
$\quad$ Capillary leak syndrome/fluid resuscitation & $45(88.2)$ & $34(94.4)$ & $<.001$ \\
\hline
\end{tabular}

Categorized risk factors between survivors and non-survivors in the high risk group ( 87 patients with $\geq 2$ categorized risk factors). ICU, intensive care unit. Data are presented as mean \pm standard deviation, unless otherwise indicated.

Table 5 General characteristics of the study cohort, and comparisons between patients

\begin{tabular}{|c|c|c|c|c|}
\hline & Study cohort & $\geq 2$ categorized risk factors & $<2$ categorized risk factors & $p$ value \\
\hline Patients ( $n$, percent) & $151(100)$ & $87(57.6)$ & $64(42.4)$ & - \\
\hline Age (years) & $56.7 \pm 17.5$ & $55.8 \pm 17.2$ & $58 \pm 17.9$ & NS \\
\hline Male gender ( $n$, percent) & $106(70.2)$ & $66(75.9)$ & $40(62.5)$ & NS \\
\hline Body mass index (kilograms per square meter) & $25.9 \pm 5.3$ & $26.3 \pm 5.6$ & $25.3 \pm 4.9$ & NS \\
\hline Acute Physiology and Chronic Health Evaluation score, points & $20.1 \pm 8.4$ & $22.8 \pm 7.9$ & $16.5 \pm 7.8$ & $<.001$ \\
\hline Sequential Organ Failure Assessment score on day 1, points & $6.4 \pm 3.9$ & $7.9 \pm 3.9$ & $4.4 \pm 2.7$ & $<.001$ \\
\hline Number of organ failures on day 1 , points & $3 \pm 1.3$ & $3.2 \pm 1.3$ & $2.2 \pm 1.1$ & $<.001$ \\
\hline Sequential Organ Failure Assessment score maximum, points & $8.1 \pm 5.2$ & $10.5 \pm 5.1$ & $4.8 \pm 3.1$ & $<.001$ \\
\hline Number of organ failures maximum, points & $3 \pm 1.5$ & $3.8 \pm 1.5$ & $2.3 \pm 1.1$ & $<.001$ \\
\hline Positive cumulative fluid balance during ICU stay & $59(39.1)$ & $34(39)$ & $25(39)$ & NS \\
\hline ICU stay (days) ${ }^{a}$ & $5(3-12)$ & $9(4-25)$ & $3(2-4)$ & $<.001$ \\
\hline Hospital stay (days) ${ }^{a}$ & $16(9-37)$ & $24(4-55)$ & $13(8-21)$ & $=.001$ \\
\hline Hospital mortality ( $n$, percent) & $42(27.8)$ & $36(41.4)$ & $6(14.3)$ & $<.001$ \\
\hline \multicolumn{5}{|l|}{ Number of categorized risk factors } \\
\hline On admission & $1.7 \pm 1.0$ & $2.4 \pm 0.9$ & $0.8 \pm 0.4$ & $<.001$ \\
\hline During ICU stay & $2.0 \pm 1.1$ & $2.8 \pm 0.8$ & $0.8 \pm 0.3$ & $<.001$ \\
\hline \multicolumn{5}{|l|}{ Risk factors during ICU stay ${ }^{\mathrm{b}}$ ( $n$, percent) } \\
\hline Diminished abdominal wall compliance & $114(75.5)$ & $83(95.4)$ & $31(48.4)$ & $<.001$ \\
\hline Mechanical ventilation & $104(68.9)$ & $77(88.5)$ & $27(42.2)$ & $<.001$ \\
\hline Head of bed $>30^{\circ}$ & $135(89.4)$ & $83(95.4)$ & $52(81.3)$ & $=.005$ \\
\hline Body mass index $\geq 30 \mathrm{~kg} / \mathrm{m}^{2}$ & $36(23.8)$ & $26(29.9)$ & $10(15.6)$ & $=.04$ \\
\hline Increased intraluminal contents & $44(29.1)$ & $44(50.6)$ & $0(0)$ & $<.001$ \\
\hline Gastroparesis & $37(24.5)$ & $37(42.5)$ & $0(0)$ & $<.001$ \\
\hline lleus & $21(13.9)$ & $21(24.1)$ & $0(0)$ & $<.001$ \\
\hline
\end{tabular}


Table 5 General characteristics of the study cohort, and comparisons between patients (Continued)

\begin{tabular}{|c|c|c|c|c|}
\hline Increased abdominal contents & $26(17.2)$ & $26(29.9)$ & $0(0)$ & $<.001$ \\
\hline Ascites/liver dysfunction & $14(9.3)$ & $14(16.1)$ & $0(0)$ & $=.001$ \\
\hline Ascites of other causes & $4(2.6)$ & $4(4.6)$ & $0(0)$ & NS \\
\hline Capillary leak syndrome/fluid resuscitation & $97(64.2)$ & $79(90.8)$ & $18(28.1)$ & $<.001$ \\
\hline Acidosis & $32(21.2)$ & $28(32.2)$ & $4(6.3)$ & $<.001$ \\
\hline Hypotension & $68(45)$ & $61(70.1)$ & $7(10.9)$ & $<.001$ \\
\hline Hypothermia & $7(4.6)$ & $7(8)$ & $0(0)$ & $=.01$ \\
\hline Multiple transfusions & $3(2)$ & $3(3.4)$ & $0(0)$ & NS \\
\hline Coagulopathy & $48(31.8)$ & $40(46)$ & $8(12.5)$ & $<.001$ \\
\hline Massive fluid resuscitation & $49(32.5)$ & $40(46)$ & $9(14.1)$ & $<.001$ \\
\hline Acute pancreatitis & $5(3.3)$ & $5(5.7)$ & $0(0)$ & NS \\
\hline Oliguria & $43(28.5)$ & $35(40.2)$ & $8(12.5)$ & $<.001$ \\
\hline Sepsis & $68(45)$ & $56(64.4)$ & 12 (18.8) & $<.001$ \\
\hline
\end{tabular}

General characteristics of the study cohort, and comparisons between patients with $\geq 2$ vs. $<2$ categorized risk factors for intra-abdominal hypertension. ${ }^{\text {a Data are }}$ presented as median and interquartile range. ${ }^{b}$ Abdominal surgery with primary fascial or tight closure, major trauma, major burns, prone positioning, colonic pseudo-obstruction, hemoperitoneum or pneumoperitoneum; other intra-abdominal injuries and damage control laparotomy are not included due to the very low number of patients with these risk factors. APACHE II, Acute Physiology and Chronic Health Evaluation; CRF, categorized risk factors; IAH, intra-abdominal hypertension; ICU, intensive care unit; NS, not significant; SOFA, Sequential Organ Failure Assessment. Data are presented as mean \pm standard deviation, unless otherwise indicated.

Table 6 General characteristics of the patients with $\geq 2$ categorized risk factors and comparisons between patients

\begin{tabular}{|c|c|c|c|c|}
\hline & $\begin{array}{l}\geq 2 \text { Categorized risk } \\
\text { factors }\end{array}$ & $\begin{array}{l}\text { Intra-abdominal } \\
\text { hypertension }\end{array}$ & $\begin{array}{l}\text { No intra-abdominal } \\
\text { hypertension }\end{array}$ & $\begin{array}{l}p \\
\text { value }\end{array}$ \\
\hline Patients ( $n$, percent) & $87(100)$ & $59(67.8)$ & $28(32.2)$ & \\
\hline Age (years) & $55.8 \pm 17.2$ & $59.3 \pm 16.4$ & $48.5 \pm 16.6$ & .006 \\
\hline Male gender ( $n$, percent) & $66(75.9)$ & $44(74.6)$ & $22(78.6)$ & NS \\
\hline $\begin{array}{l}\text { Body mass index (kilograms per square } \\
\text { meter) }\end{array}$ & $26.3 \pm 5.6$ & $27.7 \pm 5.6$ & $23.4 \pm 4.2$ & $<.001$ \\
\hline APACHE II score, points & $22.8 \pm 7.9$ & $24.4 \pm 7.8$ & $19.5 \pm 7.2$ & .007 \\
\hline SOFA score on day 1 , points & $7.9 \pm 3.9$ & $8.8 \pm 4.0$ & $6.2 \pm 3.3$ & .004 \\
\hline Number of organ failures on day 1 , points & $3.2 \pm 1.3$ & $3.5 \pm 1.3$ & $2.5 \pm 1.2$ & .002 \\
\hline SOFA score maximum, points & $10.5 \pm 5.1$ & $11.7 \pm 4.7$ & $7.9 \pm 4.9$ & .001 \\
\hline Number of organ failures maximum, points & $3.8 \pm 1.5$ & $4.2 \pm 1.4$ & $3.0 \pm 1.4$ & $<.001$ \\
\hline $\begin{array}{l}\text { Positive cumulative fluid balance during ICU } \\
\text { stay }\end{array}$ & $34(39)$ & $25(42.4)$ & $9(32.1)$ & NS \\
\hline ICU stay (days) ${ }^{a}$ & $9(4-25)$ & $11(7-29)$ & $4(3-9)$ & $<.001$ \\
\hline Hospital stay (days) ${ }^{a}$ & $24(4-55)$ & $29(13-61)$ & $15(6-34)$ & .005 \\
\hline Hospital mortality ( $n$, percent) & $36(41.4)$ & $27(45.8)$ & $9(32.1)$ & NS \\
\hline \multicolumn{5}{|l|}{ Number of categorized risk factors } \\
\hline On admission & $2.4 \pm 0.9$ & $2.5 \pm 0.9$ & $2.1 \pm 0.8$ & .05 \\
\hline During ICU stay & $2.8 \pm 0.8$ & $2.9 \pm 0.8$ & $2.4 \pm 0.6$ & .006 \\
\hline \multicolumn{5}{|l|}{ Risk factors during ICU stay ${ }^{\mathrm{b}}$ ( $n$, percent) } \\
\hline Diminished abdominal wall compliance & $83(95.4)$ & $57(96.6)$ & $26(92.9)$ & NS \\
\hline Mechanical ventilation & $77(88.5)$ & $51(86.4)$ & $26(92.9)$ & NS \\
\hline Head of bed $>30^{\circ}$ & $83(95.4)$ & $57(96.6)$ & $26(92.9)$ & NS \\
\hline Body mass index $\geq 30 \mathrm{~kg} / \mathrm{m}^{2}$ & $26(29.9)$ & $24(40.7)$ & $2(7.1)$ & .001 \\
\hline Increased intraluminal contents & $44(50.6)$ & $33(55.9)$ & $11(39.3)$ & NS \\
\hline Gastroparesis & $37(42.5)$ & $28(47.5)$ & $9(32.1)$ & NS \\
\hline lleus & $21(24.1)$ & $16(27.1)$ & $5(17.9)$ & NS \\
\hline Increased abdominal contents & $26(29.9)$ & $23(39)$ & $3(10.7)$ & .007 \\
\hline Ascites/liver dysfunction & $14(16.1)$ & $12(20.3)$ & $2(7.1)$ & NS \\
\hline Ascites of other causes & $4(4.6)$ & $4(6.8)$ & $0(0)$ & NS \\
\hline
\end{tabular}


Table 6 General characteristics of the patients with ???2 categorized risk factors and comparisons between patients (Continued)

\begin{tabular}{|c|c|c|c|c|}
\hline $\begin{array}{l}\text { Capillary leak syndrome/fluid } \\
\text { resuscitation }\end{array}$ & $79(90.8)$ & $55(93.2)$ & $24(85.7)$ & NS \\
\hline Acidosis & $28(32.2)$ & $22(37.3)$ & $6(21.4)$ & NS \\
\hline Hypotension & $61(70.1)$ & $46(78)$ & 15 (53.6) & .02 \\
\hline Hypothermia & 7 (8) & $5(8.5)$ & $2(7.1)$ & NS \\
\hline Multiple transfusions & $3(3.4)$ & $2(3.4)$ & $1(3.6)$ & NS \\
\hline Coagulopathy & $40(45.9)$ & $30(50.8)$ & $10(35.7)$ & NS \\
\hline Massive fluid resuscitation & $40(46)$ & $30(50.8)$ & $10(35.7)$ & NS \\
\hline Acute pancreatitis & $5(5.7)$ & $5(8.5)$ & $0(0)$ & .005 \\
\hline Oliguria & $35(40.2)$ & $27(45.8)$ & 8 (28.6) & NS \\
\hline Sepsis & $56(64.4)$ & $40(67.8)$ & $16(57.1)$ & NS \\
\hline IAP during ICU stay & $12 \pm 3$ & $13 \pm 1$ & $8 \pm 1$ & $<.001$ \\
\hline APP mean during ICU stay & $74 \pm 13$ & $73 \pm 12$ & $74 \pm 15$ & NS \\
\hline
\end{tabular}

General characteristics of the patients with $\geq 2$ categorized risk factors, and comparisons between patients with and without intra-abdominal hypertension. ${ }^{\mathrm{a}}$ Data are presented as median and interquartile range. ${ }^{b}$ Abdominal surgery with primary fascial or tight closure, major trauma, major burns, prone positioning, colonic pseudo-obstruction, hemoperitoneum or pneumoperitoneum; other intra-abdominal injuries and damage control laparotomy are not included due to the very low number of patients with these risk factors. APACHE II, APP, abdominal perfusion pressure; Acute Physiology and Chronic Health Evaluation; CRF, categorized risk factors; IAH, intra-abdominal hypertension; IAP, intra-abdominal pressure (mmHg); ICU, intensive care unit; NS, not significant; SOFA, Sequential Organ Failure Assessment. Data are presented as mean \pm standard deviation, unless otherwise indicated.

Table 7 Comparison of organ failure according to SOFA score between IAH and non-IAH patients

\begin{tabular}{|c|c|c|c|}
\hline & Intra-abdominal hypertension $(n=59)$ & No intra-abdominal hypertension $(n=28)$ & $p$ value \\
\hline \multicolumn{4}{|c|}{ Organ failures on day 1} \\
\hline Respiratory & $56(94.9)$ & $27(96.5)$ & NS \\
\hline Circulatory & $43(72.8)$ & $11(39.2)$ & $<.003$ \\
\hline Renal & $32(54.2)$ & $4(14.6)$ & $<.001$ \\
\hline Hepatic & $21(35.6)$ & $6(21.4)$ & NS \\
\hline Neurological & $28(47.5)$ & $16(67.1)$ & NS \\
\hline Coagulation & $28(47.5)$ & $7(25)$ & $=.04$ \\
\hline \multicolumn{4}{|c|}{ Organ failures during ICU stay } \\
\hline Respiratory & $58(98.4)$ & $26(92.8 \%)$ & NS \\
\hline Circulatory & $47(79.7)$ & $16(57.1 \%)$ & $=.02$ \\
\hline Renal & $38(64.4)$ & $6(21.5 \%)$ & $<.001$ \\
\hline Hepatic & $32(54.3)$ & $8(28.5 \%)$ & $=.02$ \\
\hline Neurologic & $37(62.7)$ & $18(6.3 \%)$ & NS \\
\hline Coagulation & $37(62.7)$ & 11 (3.3\%) & $=.04$ \\
\hline
\end{tabular}

Comparison of organ failure according to SOFA score (Sequential Organ Failure Assessment Score) between IAH and non-IAH patients on admission and during the entire ICU stay. IAH, intra-abdominal hypertension; ICU, intensive care unit; NS, not significant. Values are $n$ (percent).

Table 8 Characteristics of patients with intra-abdominal hypertension and comparisons between survivors and nonsurvivors

\begin{tabular}{lcccc}
\hline & Intra-abdominal hypertension & Survivors & Non-survivors & $\boldsymbol{p}$ value \\
\hline Patients $(n$, percent) & $59(100)$ & $32(54.2)$ & $27(45.8)$ & - \\
Age (years) & $59.3 \pm 16.4$ & $55.7 \pm 16.6$ & $63.5 \pm 15.5$ & $\mathrm{NS}$ \\
Male gender $(n$, percent) & $44(74.6)$ & $23(71.8 \%)$ & $21(77.8)$ & $\mathrm{NS}$ \\
Body mass index (kilogram per square meter) & $27.7 \pm 5.6$ & $27.8 \pm 6.8$ & $27.6 \pm 3.9$ & $\mathrm{NS}$ \\
APACHE II score, points & $24.4 \pm 7.8$ & $22.5 \pm 8.8$ & $26.6 \pm 5.7$ & .03 \\
SOFA score on day 1, points & $8.8 \pm 4$ & $7.5 \pm 3.7$ & $10.2 \pm 3.9$ & .009 \\
Number of organ failures on day 1, points & $1.7 \pm 1.1$ & $1.3 \pm 1$ & $2.2 \pm 0.9$ & $<.001$ \\
SOFA score maximum, points & $11.7 \pm 4.7$ & $10.2 \pm 4.2$ & $13.5 \pm 4.8$ & .006 \\
Number of organ failures maximum & $2.6 \pm 1.3$ & $2.3 \pm 1.2$ & $3.1 \pm 1.3$ & .02 \\
Number of categorized risk factors & $2.5 \pm 0.9$ & $2.3 \pm 0.8$ & $2.8 \pm 0.9$ & 03
\end{tabular}


Table 8 Characteristics of patients with intra-abdominal hypertension and comparisons between survivors and nonsurvivors (Continued)

\begin{tabular}{|c|c|c|c|c|}
\hline At admission & $2.9 \pm 0.8$ & $2.7 \pm 0.8$ & $3.3 \pm 0.7$ & .003 \\
\hline During ICU stay & $5(8.5)$ & $3(9.4)$ & $2(7.4)$ & - \\
\hline \multicolumn{5}{|l|}{ Classification of IAH according to } \\
\hline \multicolumn{5}{|c|}{ Presentation or development of symptoms ( $n$, percent) } \\
\hline Acute & $51(86.4)$ & $26(81.2)$ & $25(92.6)$ & - \\
\hline Subacute & $3(5.1)$ & $3(9.4)$ & $0(0)$ & NS \\
\hline Chronic & $6(10.2)$ & $3(9.4)$ & $3(11.1)$ & NS \\
\hline \multicolumn{5}{|l|}{ Grade of IAP reached during ICU } \\
\hline \multicolumn{5}{|l|}{ stay ( $n$, percent) } \\
\hline I (12-15 mmHg) & $34(57.6)$ & $19(59.4)$ & $15(55.5)$ & NS \\
\hline II (16-20 mmHg) & $14(23.7)$ & $8(25)$ & $6(22.2)$ & NS \\
\hline III (21-25 mmHg) & $5(8.5)$ & $2(6.2)$ & $3(11.1)$ & NS \\
\hline IV $(>25 \mathrm{mmHg})$ & $13 \pm 1$ & $14 \pm 2$ & $13 \pm 1$ & NS \\
\hline IAP mean during ICU stay & $73 \pm 12$ & $74 \pm 12$ & $71 \pm 12$ & .001 \\
\hline APP mean during ICU stay & $35(59.3)$ & $21(65.6)$ & $14(51.8)$ & NS \\
\hline \multicolumn{5}{|l|}{ Time of IAH development ( $n$, percent) } \\
\hline On admission & $24(40.7)$ & $11(34.4)$ & $13(48.1)$ & NS \\
\hline During ICU stay & $1(1-3)$ & $1(1-3)$ & $1(1-4)$ & NS \\
\hline Day of IAH development during ICU stay ${ }^{a}$ & $8(4-16)$ & $7(4-16)$ & $9(6-16)$ & NS \\
\hline Days with maintained IAH during ICU stay ${ }^{a}$ & $34(57.6)$ & $12(37.5)$ & $22(81.5)$ & \\
\hline \multicolumn{5}{|l|}{ Non-resolved IAH ( $n$, percent) } \\
\hline Positive cumulative fluid balance at IAH diagnosis & $49(83)$ & $27(84.4)$ & $22(81.5)$ & \\
\hline Positive cumulative fluid balance during ICU stay & $25(42.4)$ & $10(31.2)$ & $15(55.5)$ & \\
\hline ICU stay (days) ${ }^{\mathrm{a}}$ & $1(7-29)$ & $12(7-26)$ & $12(6-33)$ & \\
\hline Hospital stay after ICU (days) ${ }^{a}$ & $29(13-61)$ & $34(23-58)$ & $16(8-65)$ & \\
\hline
\end{tabular}

${ }^{2}$ Data are presented as median and interquartile range. APACHE II, Acute Physiology and Chronic Health Evaluation; APP, abdominal perfusion pressure; IAH, intra-abdominal hypertension; IAP, intra-abdominal pressure (mmHg); ICU, intensive care unit; NS, not significant; SOFA, Sequential Organ Failure Assessment. Data are presented as mean \pm standard deviation, unless specified.

\section{Discussion}

This is a prospective epidemiologic study performed in a population of exclusive critically ill medical patients following the WSACS guidelines for the assessment of IAH (screening, IAP measurement, definitions, and classification recommendations). Other published studies had been performed in mixed populations (medical and surgical), before the WSACS consensus statement, based on different definitions of IAH or limited by incomplete assessment in terms of screening or classification of the IAH [17-21].

Our study revealed several interesting findings. Firstly, there was a large number of patients with high risk $(\geq 2$ $\mathrm{CRF}$ ) for IAH development, and the incidence of IAH in this group was high; in addition, the mortality in this subgroup was significantly higher as compared with the low risk subgroup. Secondly, there was an association of IAH with illness severity and the number/type of RF reported in the literature. Thirdly, IAH was a predictor of mortality in association with other clinical factors. And finally, nonresolution of IAH was an independent predictive factor of mortality. Our results support the importance of implementing screening and assessment protocols, according to
WSACS recommendation, in order to identify a subgroup of high risk patients with high mortality and high incidence of IAH whom should be monitored for IAP.

\section{Screening, risk factors and incidence of IAH}

A large number of patients presented $\geq 2$ CRF for IAH development, and the incidence of IAH in this group was high $(67.8 \%)$ on admission or during the ICU stay. In addition, several high risk patients without IAH might have shown underestimated IAP levels because of the different factors usually present in the critically ill population, such as sedation or gastric/colonic decompression. A high incidence of IAH had also been reported in the literature [17-21]; however, when trying to combine our results with previously described findings, three main methodology issues should be accounted for: a) incidence is recorded on admission or during the first week; b) most reports refer to mixed populations (both medical and surgical); c) definitions of IAH differed across the studies (incidence rates can vary depending on the threshold IAP level used as a diagnostic criterion). Unlike previous studies, our study analyzes the incidence of IAH in a high risk population on 


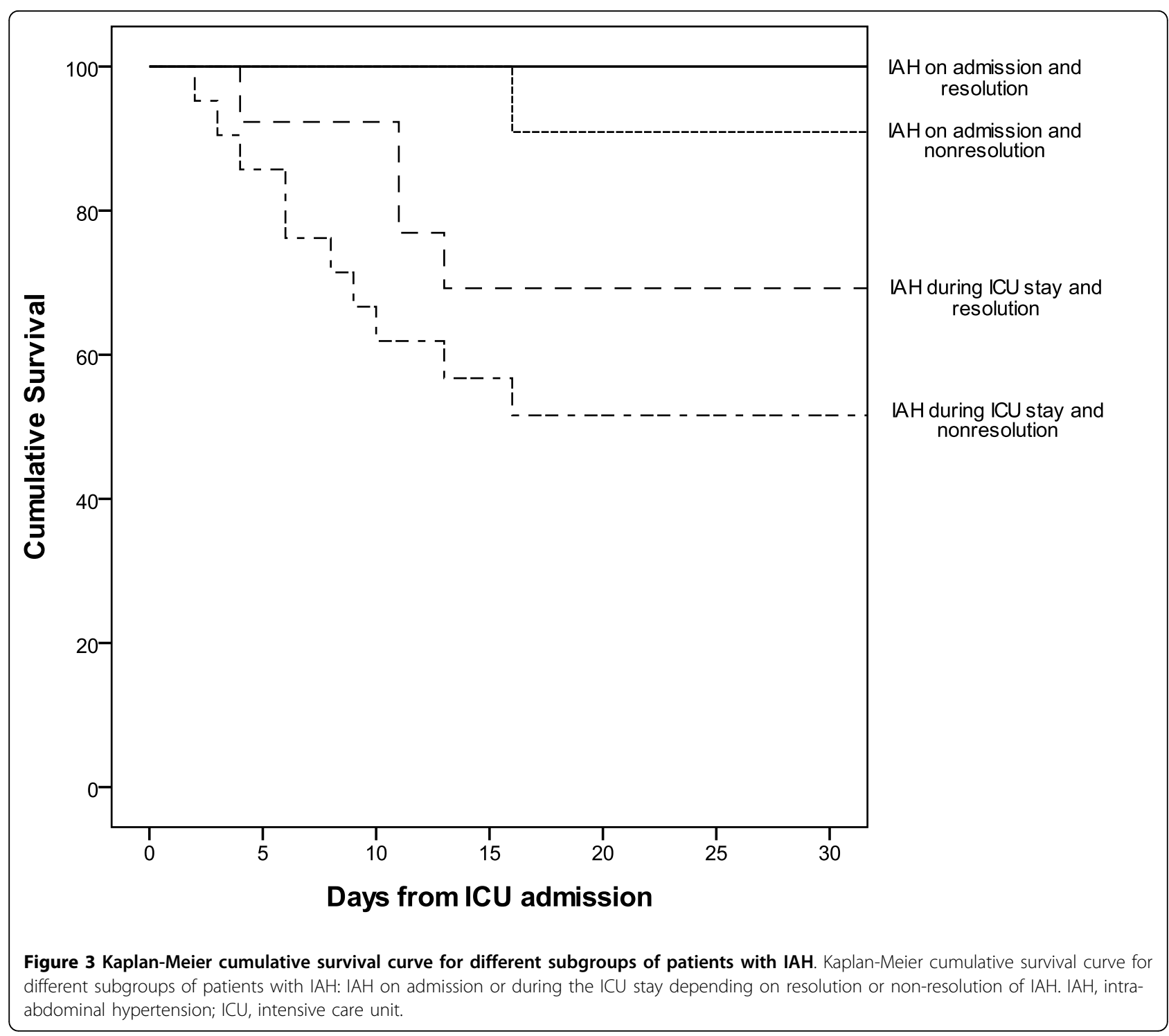

admission and throughout the entire ICU stay, following WSACS consensus guidelines, and exclusively in critically ill medical patients. Reintam et al. [17] and Vidal et al. [18] have also used WSACS definitions for IAH, and they report incidence rates of $37 \%$ in a mixed population, and $43 \%$ in the medical subgroup of a mixed population, respectively.

Numerous conditions predispose to IAH development, and our results are consistent with those from other studies [20,21]. In the high risk group, we found an

Table 9 Logistic regression model for predictors of development of IAH in the high risk group

\begin{tabular}{|c|c|c|c|c|}
\hline & Coefficients & Odds ratio & $95 \%$ confidence interval & $p$ value \\
\hline Age $\geq 65$ years & 0.780 & 2.18 & $0.66-7.15$ & .19 \\
\hline Body mass index $\geq 30, \mathrm{~kg} / \mathrm{m}^{2}$ & 2.312 & 10.09 & $1.97-51.58$ & .005 \\
\hline SOFA score at admission & 0.153 & 1.16 & $1.00-1.35$ & .04 \\
\hline Number of categorized risk factors for IAH during ICU stay & 0.840 & 2.31 & $1.09-4.88$ & .02 \\
\hline Constant & -3.408 & & & \\
\hline
\end{tabular}

Predictors of development of IAH in the group with $\geq 2$ categorized risk factors for IAH (87 patients). Cl, confidence interval; SOFA, Sequential Organ Failure Assessment; IAH, intra-abdominal hypertension; ICU, intensive care unit. Area under receiver operating characteristic curve $=0.80$; Hosmer-Lemeshow goodnessof-fit $\left(x^{2}=4.22 ; p=0.835\right)$. 
Table 10 Logistic regression model for predictors of mortality in the high risk group

\begin{tabular}{|c|c|c|c|c|}
\hline & Coefficients & Odds ratio & 95\% Confidence interval & $p$ value \\
\hline Age $\geq 65$ yrs & 2.057 & 3.82 & $2.08-29.28$ & .002 \\
\hline APACHE $\|$ score $\geq 20$ & 1.614 & 5.02 & $1.11-22.70$ & .03 \\
\hline SOFA score maximum during ICU stay & 0.224 & 1.25 & $1.03-1.51$ & .01 \\
\hline Positive cumulative fluid balance during ICU stay & 1.730 & 5.64 & $1.50-21.14$ & .06 \\
\hline No. of categorized risk factors for IAH during ICU stay & 1.200 & 3.32 & $0.94-11.72$ & .34 \\
\hline Increased abdominal content during ICU stay & 1.007 & 2.73 & $0.34-21.78$ & .08 \\
\hline $\mathrm{IAH}$ & 1.409 & 4.09 & $0.83-20.12$ & \\
\hline Constant & -9.908 & & & \\
\hline
\end{tabular}

Predictors of mortality in the group with $\geq 2$ categorized risk factors for IAH (87 patients). APACHE II, Acute Physiology and Chronic Health Evaluation; Cl, confidence interval; IAH, intra-abdominal hypertension; ICU, intensive care unit; SOFA, Sequential Organ Failure Assessment. Area under receiver operating characteristic curve $=0.78$; Hosmer-Lemeshow goodness-of-fit $(x 2=3.78 ; p=0.870)$.

association between IAH and the CRF proposed by the WSACS, specially the increased abdominal content. Obesity and the number of CRF for IAH during ICU stay were independent predictors of IAH development. Data on fluid balance were only qualitative (positive or negative), and the association between IAH and massive fluid loading in the presence of sepsis and/or capillary leak described in the literature could not be studied.

\section{Severity scores and organ failure in patients with IAH}

Increases in IAP have deleterious effects on end-organ system function by compromising perfusion, thus leading to multiorganic dysfunction (MOD) and poor outcome. In our population, IAH was significantly associated with higher severity of illness scores and incidence of organ failure. In addition, the study design allowed us to compare organ impairment during ICU stay between patients with and without IAH (Table 5). Previous studies analyzed organ impairment only during the first days of ICU stay, thus making it difficult to study the association between MOD and IAH throughout the entire ICU stay.

\section{Characteristics of IAH}

WSACS gives special attention to the IAH classification; this correlates with the patient's profile, namely, whether the patient is medical or surgical [17-21,32-34]. Classification of IAH as acute and with high IAP grades (IIIIV) is typical for surgical patients, whereas classification of IAH as subacute and with low-moderate IAP grades
(I-II) is characteristic for medical patients [17-21,35]. Our results in medical patients are consistent with this observation. The IAH classification varies depending on the study; however, no studies include all the classification types currently recommended by WSACS. In summary, a complete classification is important for planning future studies and comparing results, and the differentiation between medical or surgical patients is, in our opinion, necessary for a correct analysis based on the differences with regard to the causes and evolution of $\mathrm{IAH}$ in those two types of patient's profile.

Fourteen patients reached grade III of IAH, and five grade IV, but none of them developed ACS because a sustained elevation of IAP $>20 \mathrm{mmHg}$ associated with organ deterioration was not detected. In concordance with this, no patient required surgical abdominal decompression.

\section{Prognostic implications of IAH}

Development of IAH has been described as an independent predictor of mortality in mixed populations [20]. In our study IAH was a non-independent predictor of mortality, and this supports the fact that IAH was a marker of mortality in association with other clinical factors. In accordance with this, patients with IAH had higher severity scores and number of CRF for IAH that can independently increase mortality risk. In fact, the number of CRF during ICU stay was an independent predictor of mortality in the group with IAH.

Table 11 Logistic regression model for predictors of mortality in the IAH group

\begin{tabular}{|c|c|c|c|c|}
\hline & Coefficients & Odds ratio & 95\% confidence interval & $p$ value \\
\hline Age $\geq 65$ yrs & 2.811 & 16.63 & $2.46-112.30$ & .004 \\
\hline SOFA score maximum during ICU stay & 0.196 & 1.22 & $0.97-1.53$ & .09 \\
\hline No. of categorized risk factors for IAH during ICU stay & 2.297 & 9.94 & $1.59-62.00$ & .01 \\
\hline Increased abdominal content during ICU stay & 1.847 & 6.34 & $0.43-93.31$ & .17 \\
\hline Non-resolution of IAH & 2.577 & 13.15 & 22.13-81.92 & .006 \\
\hline Constant & -13.953 & & & \\
\hline
\end{tabular}

Predictors of mortality in the patients with IAH (59 patients). Cl, confidence interval; IAH, intra-abdominal hypertension; ICU, intensive care unit; SOFA, Sequential Organ Failure Assessment. Area under receiver operating characteristic curve $=0.80$ 
We found a high rate of resolution of IAH in our medical patients, but non-resolution was an independent predictor of mortality, resulting in a considerably high OR (non-resolution meant that death was 13 times more likely). Treatment of IAH was nonsurgical in all cases and none developed ACS, although no conclusions can be drawn in the absence of clinical intervention data. Management guidelines for IAH have been proposed by the WSACS [24] although they are based on studies that analyze therapeutic strategies in isolation and in different patient profiles [36-40].

\section{Limitations of the study}

IAP was not measured in all cases (only in those with $\geq 2$ CRF), and patients with $<2 \mathrm{CRF}$ and no IAP recorded might have developed IAH during their ICU stay (e.g., patients with mechanical ventilation and therefore, diminished abdominal wall compliance but with no RF in other categories and no IAP measured). Therefore, the study followed the WSACS screening recommendations but was not designed to validate them in order to identify a subgroup of high risk patients for IAH development, as compared with a low risk subgroup. The study was underpowered to analyze each RF of the four categories as predictors of IAH (small sample size) or the IAP level as a predictor of mortality (the IAP $_{\text {mean }}$ in the patients with IAH were relatively low to perform comparisons in the outcome). Not all patients who had their IAP measured were sedated, which could have caused a falsely elevated IAP. Although resolution of IAH was a prognostic factor, our study was not designed to focus on therapeutic management of IAH, and no conclusions can be drawn or ascertainments can be made about the strategies that were effective.

\section{Conclusions}

Critically ill medical patients admitted to the ICU with high risk of IAH development ( $\geq 2 \mathrm{CRF}$ ) have high morbidity, mortality, and incidence of IAH, so IAP should be measured as recommended by the WSACS. In this subgroup of patients, the presence of IAH is associated with mortality as a non-independent risk factor, and non-resolution of this process is an independent predictor of mortality. Our study supports the implementation of protocols based on WSACS guidelines for IAH screening and assessment to allow an early approach to this syndrome. Large, multicenter randomized controlled clinical trials are needed to confirm our results and to further promote the WSACS recommendations regarding IAH screening and management.

\section{Consent}

Written informed consent was obtained from the patients or their relatives for publication of this case report. A copy of the written consent is available for review by the Editor-in-Chief of this journal.

\section{Abbreviations}

APACHE II: Acute Physiology and Chronic Health Evaluation; APP: abdominal perfusion pressure; BMl: body mass index; CRF: categorized risk factors; $I \mathrm{AH}$ : intra-abdominal hypertension; IAP: intra-abdominal pressure; ICU: intensive care unit; MOD: multiple organ dysfunction; RF: risk factors; SOFA: Sequential Organ Failure Assessment; WSACS: World Society of Abdominal Compartment Syndrome.

\section{Acknowledgements}

This article has been published as part of Annals of Intensive Care Volume 2 Supplement 1, 2012: Diagnosis and management of intra-abdominal hypertension and abdominal compartment syndrome. The full contents of the supplement are available online at http://www.annalsofintensivecare. com/supplements/2/S1

We are indebted to the study coordinators, clinical research associates, and intensive care residents. We are especially grateful to all the nurses and nursing assistants in our ICU for IAP measurements. We are indebted to the Fundación para la Investigación Biomédica del Hospital Gregorio Marañón, especially Mr Thomas O’Boyle and Dr Jose María de Miguel, for advice in the preparation of this manuscript, and to the faculty members of the WSACS for raising concern about intra-abdominal hypertension in intensive care patients.

\section{Author details}

${ }^{1}$ Intensive Care Unit, Hospital General Universitario Gregorio Marañón, Calle Doctor Esquerdo 46, Madrid, 28007, Spain. ²Department of Internal Medicine, Hospital General Universitario Gregorio Marañón, Calle Doctor Esquerdo 46, Madrid, 28007, Spain.

\section{Authors' contributions}

All authors read and approved the final manuscript. PS conceived the study and participated in its design and coordination. PS and JM performed the statistical analysis. LA and PG participated in analysis and interpretation of data. IM, MZ and MT collected data. All authors contributed to the writing and preparation of the manuscript.

\section{Authors' information}

PS, MD, PhD; JM, MD, Associate Professor of Medicine, Universidad Complutense de Madrid (UCM), Spain; MT, RN, Chief of Critical Care Nurse Department; IM, MD; MZ, MD; LA, MD, PhD, Chief of Internal Medicine Department, Professor of Medicine, UCM, Spain; PG, MD.

\section{Competing interests}

The authors declare that they have no competing interests.

Published: 5 July 2012

\section{References}

1. Balogh Z, McKinley BA, Cox CS Jr, Allen SJ, Cocanour CS, Kozar RA, Moore EE, Miller CC III, Weisbrodt NW, Moore FA: Abdominal compartment syndrome: the cause of effect of postinjury multiple organ failure. Shock 2003, 20(6):483-492.

2. Balogh Z, McKinley BA, Holcomb JB, Miller CC, Cocanour CS, Kozar RA, Valdivia A, Ware DN, Moore FA: Both primary and secondary abdominal compartment syndrome can be predicted early and are harbingers of multiple organ failure. J Trauma 2003, 54(5):848-859.

3. Cullen DJ, Coyle JP, Teplick R, Long MC: Cardiovascular, pulmonary, and renal effects of massively increased intra-abdominal pressure in critically ill patients. Crit Care Med 1989, 17(2):118-121.

4. Kashtan J, Green JF, Parsons EQ, Holcroft JW: Hemodynamic effects of increased abdominal pressure. J Surg Res 1981, 30(3):249-255.

5. Barnes GE, Laine GA, Giam PY, Smith EE, Granger HJ: Cardiovascular responses to elevation of intra-abdominal pressure. Am J Physiol 1985, 248(2 Pt 2):R208-13.

6. Obeid F, Saba A, Fath J, Guslits B, Chung R, Sorensen V, Buck J, Horst M: Increases in intra-abdominal pressure affect pulmonary compliance. Arch Surg 1995, 130(5):544-547. 
7. Doty JM, Saggi BH, Blocher CR, Fakhry I, Gehr T, Sica D, Sugerman HJ: Effects of increased renal parenchymal pressure on renal function. $J$ Trauma 2000, 48(5):874-877.

8. Sugrue $M$, Balogh Z, Malbrain M: Intra-abdominal hypertension and renal failure. ANZ J Surg 2004, 74(1-2):78

9. Diebel LN, Wilson RF, Dulchavsky SA, Saxe J: Effect of increased intraabdominal pressure on hepatic arterial, portal venous and hepatic microcirculatory blood flow. J Trauma 1992, 33(2):279-282.

10. Bloomfield GL, Ridings PC, Blocher CR, Marmarou A, Sugerman HJ: A proposed relationship between increased intra-abdominal, intrathoracic, and intracranial pressure. Crit Care Med 1997, 25(3):496-503.

11. Citerio G, Vascotto E, Villa F, Celotti S, Pesenti A: Induced abdominal compartment syndrome increases intracranial pressure in neurotrauma patients: a prospective study. Crit Care Med 2001, 29(7):1466-1471.

12. Raeburn CD, Moore EE, Biffl WL, Johnson JL, Meldrum DR, Offner PJ, Franciose RJ, Burch JM: The abdominal compartment syndrome is a morbid complication of postinjury damage control surgery. Am J Surg 2001, 182(6):542-546

13. Mcnelis J, Soffer S, Marini CP, Jurkiewicz A, Ritter G, Simms HH, Nathan I: Abdominal compartment syndrome in the surgical intensive care unit. Am Surg 2002, 68(1):18-23.

14. Maxwell RA, Fabian TC, Croce MA, Davis KA: Secondary abdominal compartment syndrome: an underappreciated manifestation of severe hemorrhagic shock. J Trauma 1999, 47(6):995-999.

15. Mayberry JC, Welker KJ, Goldman RK, Mullins RJ: Mechanism of acute ascites formation after trauma resuscitation. Arch Surg 2003, 138(7):773-776.

16. Balogh Z, McKinley BA, Cocanour CS, Kozar RA, Valdivia A, Sailors RM, Moore FA: Supranormal trauma resuscitation causes more cases of abdominal compartment syndrome. Arch Surg 2003, 138(6):637-642.

17. Reintman A, Parm P, Kitus R, Kern H, Starkopf J: Primary and secondary intra-abdominal hypertension-different impact on ICU outcome. Crit Care Med 2008, 34(9):1624-1631.

18. Vidal MG, Ruiz Weisser J, Gonzalez F, Toro MA, Loudet C, Balasini C, Canales H, Reina R, Estenssoro E: Incidence and clinical effects of intraabdominal hypertension in critically ill patients. Crit Care Med 2008, 36(6):1823-1831.

19. Lonardo M, Piazza O, Marco G, De Robertis E, Servillo G, Tufano R: Intraabdominal hypertension is not reliable as an early predictor of mortality in the intensive care unit. Minerva Anestesiol 2007, 73(9):447-450

20. Malbrain ML, Chiumello D, Pelosi P, Bihari D, Innes R, Ranieri VM, Del Turco M, Wilmer A, Brienza N, Malcangi V, Cohen J, Japiassu A, De Keulenaer BL, Daelemans R, Jacquet L, Laterre PF, Frank G, de Souza P, Cesana B, Gattinoni L: Incidence and prognosis of intraabdominal hypertension in a mixed population of critically ill patients: a multiplecenter epidemiological study. Crit Care Med 2005, 33(2):315-322.

21. Malbrain ML, Chiumello D, Pelosi P, Wilmer A, Brienza N, Malcangi V, Bihari D, Innes R, Cohen J, Singer P, Japiassu A, Kurtop E, De Keulenaer BL, Daelemans R, Del Turco M, Cosimini P, Ranieri M, Jacquet L, Laterre PF, Gattinoni L: Prevalence of intra-abdominal hypertension in critically ill patients: a multicentre epidemiological study. Intensive Care Med 2004, 30(5):822-829.

22. Malbrain ML, De Laet I: AIDS is coming to your ICU: be prepared for acute bowel injury and acute intestinal distress syndrome. Intensive Care Med 2008, 34(9):1565-1569.

23. Malbrain ML, Cheatham ML, Kirkpatrick A, Sugrue M, Parr M, De Waele J, Balogh Z, Leppäniemi A, Olvera C, Ivatury R, D'Amours S, Wendon J, Hillman K, Johansson K, Kolkman K, Wilmer A: Results from the International Conference of Experts on Intra-abdominal Hypertension and Abdominal Compartment Syndrome. I. Definitions. Intensive Care Med 2006, 32(11):1722-1732.

24. Cheatham ML, Malbrain ML, Kirkpatrick A, Sugrue M, Parr M, De Waele J, Balogh Z, Leppäniemi A, Olvera C, Ivatury R, D'Amours S, Wendon J, Hillman K, Wilmer A: Results from the international Conference of Experts on Intra-abdominal Hypertension and Abdominal Compartment Syndrome. II. Recommendations. Intensive Care Med 2007, 33(6):951-962.

25. Garrow JS: Quetelet index as indicator of obesity. Lancet 1986, 24;1(8491):1219

26. Knaus WA, Draper EA, Wagner DP, Zimmerman JE: APACHE II: A severity of disease classification system. Crit Care Med 1985, 13(10):818-829.
27. Vincent JL, Moreno R, Takala J, Willatts S, De Mendonça A, Bruining H, Reinhart CK, Suter PM, Thijs LG: The SOFA (Sepsis-related Organ Failure Assessment) score to describe organ dysfunction/failure. On behalf of the Working Group on Sepsis-Related Problems of the European Society of Intensive Care Medicine. Intensive Care Med 1996, 22(7):707-710.

28. Cheatham ML, Safcsak K: Intraabdominal pressure: A revised method for measurement. J Am Coll Surg 1998, 186(5):594-595.

29. Malbrain ML: Different techniques to measure intra-abdominal pressure (IAP): Time for a critical reappraisal. Intensive Care Med 2004, 30(3):357-371.

30. Cheatham ML, Vhite MW, Sagraves SG, Johnson JL, Block EF: Abdominal perfusion pressure: A superior parameter in the assessment of intraabdominal hypertension. J Trauma 2000, 49(4):621-627.

31. Malbrain ML: Abdominal perfusion pressure as prognostic marker in intra-abdominal hypertension. In Yearbook of Intensive Care and Emergency Medicine. Springer, Berlin Heidelberg New York;Vincent UL 2002:792-814.

32. Sugrue $M$, Buist MD, Hourihan F, Deane $S$, Bauman A, Hillman K: Prospective study of intra-abdominal hypertension and renal function after laparotomy. Br J Surg 1995, 82(2):235-238.

33. Ivatury RR, Porter JM, Simon RJ, Islam S, John R, Stahl WM: Intra-abdominal hypertension after life-threatening penetrating abdominal trauma: prophylaxis, incidence, and clinical relevance to gastric mucosal $\mathrm{pH}$ and abdominal compartment syndrome. J Trauma 1998, 44(6):1016-1021.

34. De Waele JJ, Hoste EA, Malbrain M: Decompressive laparotomy for abdominal compartment syndrome - a critical analysis. Crit Care Med 2006, 10(2):R51.

35. Malbrain ML: Abdominal pressure in the critically ill: measurement and clinical relevance. Intensive Care Med 1999, 25(12):1453-1458.

36. Balogh Z, Moore FA, Goettler CE, Rotondo MF, Schwab CW, Kaplan MJ: Surgical management of abdominal compartment syndrome. In Abdominal compartment syndrome. Landes Biomedical: Georgetown;lvatury RR, Cheatham ML, Malbrain MLNG, Sugrue M 2006:266-296.

37. De Waele J, Delaet I, Hoste E: The effect of neuromuscular blockers on intraabdominal pressure. Abstracts of the 36th Critical Care Congress, Orlando, Florida, USA, February 17-21, 2007. Crit Care Med 2006, 34(12 Abstr Suppl):A70.

38. Van der Spoel Jl, Oudemans-van Straaten HM, Stoutenbeek CP, Bosman RJ, Zandstra DF: Neostigmine resolves critical illness-related colonic ileus in intensive care patients with multiple organ failure. A prospective, double-blind, placebo-controlled trial. Intensive Care Med 2001, 27(5):822-827.

39. Reckard JM, Chung MH, Varma MK, Zagorski SM: Management of intraabdominal hypertension by percutaneous catheter drainage. I Vasc Interv Radiol 2005, 16(7):1019-1021.

40. Parra MW, Al-Khayat H, Smith HG, Cheatham ML: Paracentesis for resuscitation-induced abdominal compartment syndrome: an alternative to decompressive laparotomy in the burn patient. J Trauma 2006, 60(5):1119-1121.

doi:10.1186/2110-5820-2-S1-S3

Cite this article as: Santa-Teresa et al:: Incidence and prognosis of intraabdominal hypertension in critically ill medical patients: a prospective epidemiological study. Annals of Intensive Care 2012 2(Suppl 1):S3.

\section{Submit your manuscript to a SpringerOpen ${ }^{\circ}$ journal and benefit from:}

- Convenient online submission

- Rigorous peer review

- Immediate publication on acceptance

- Open access: articles freely available online

- High visibility within the field

- Retaining the copyright to your article

Submit your next manuscript at $>$ springeropen.com 